\title{
CORRECTIONAL INFLUENCE OF THE SET ON PROBABILISTIC JUDGMENT
}

\author{
Charkviani D. (Grigol Robakidze University, Tbilisi, Georgia) \\ davidcharkviani@gmail.com

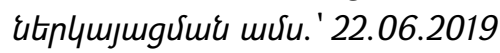

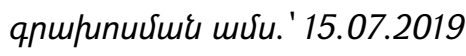

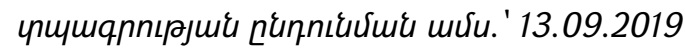

Relevant investigations of psychical mechanisms of probabilistic thinking revealed its bounded rationality reflecting of the specific of subjective comprehension of one other problem by the people. Such cognitive activity of the people plays an important role in the assessment of multiple alternatives and serves to satisfaction their different needs. Considering the main exploratory principles of General Set's Theory, we assume that functioning of probabilistic judgments determined by relevant set formed in the process of purposive activity of the people. Take into consideration of temporary parameter two forms of the set defined: prospective (orientation on the results of future events) and situational (orientation on results of current events). Obtained experimental data showed: prospective set, compared with situational set, made significant corrective influence on erroneous probabilistic judgments. It indicates the importance of clear cognitive fixation by the subjects of instrumental dependence between primary and secondary expected results.

Keywords: probabilistic judgment, prospective set, situational set, corrective influence, judgment confidence, feedback.

Relevant investigations of psychical mechanisms of probabilistic thinking revealed its bounded rationality reflecting of the specific of subjective comprehension of one other problem by the people. Such cognitive activity of the people plays an important role in the assessment of multiple alternatives and serves to satisfaction their different needs. Considering the main exploratory principles of General Set's Theory, we assume that functioning of probabilistic judgments determined by relevant set formed in the process of purposive activity of the people. Take into consideration of temporary parameter two forms of the set defined: prospective (orientation on the results of future events) and situational (orientation on results of current events). Obtained experimental data showed: prospective set, compared with situational set, made significant corrective influence on erroneous probabilistic judgments. It indicates the importance of clear cognitive fixation by the subjects of instrumental dependence between primary and secondary expected results.

Keywords: probabilistic judgment, prospective set, situational set, corrective influence, judgment confidence, feedback. 
In General Theory of the Set together with unconscious processes, the property of reasonability is underlined. In determination of molar behavior, the decisive role is derived to consciousness. Knowledge acquisition by students in high institutions is an evident example of task-oriented behavior. This process means student's oriented comprehension of specific of future profession, acquisition of professional skills, which should form subjects by students is done on the basis of task-oriented activity. They have one common goal: to finish the study and get corresponding certificate. Besides, achievement of this final goal is possible only with achievement of intermediate goals (count of weekly seminars, monthly tests indexes and total examination marks on different subjects for definite periods of the time). This process requires oriented activity from students, meaning adequate consciousness of instrumental significance of intermediate goals for achievement of common expected goal. Taking into account temporal parameter, two forms of the set can be indicated: prospective (orientation on the result of future event) and situational (orientation on current event). Thus, the taskoriented behavior is the process, in which by means of situational end prospective sets in views, step by step achievement of preliminary results short and longtime occurs (that is the instrumental meaning), which at least provides achievement final result. Besides, being the sets oriented on the solution of one and the same problem, they possess also the property of intentionality.

Theoretical and experimental research the peculiarities of thinking were conducted by Georgian psychologists. The subject of the investigations were such essential properties of thinking as the process of generalization, subjective comprehension and corresponding denomination. The obtained data are important for detection and description of psychic mechanisms, determining formation and functioning of everyday concepts. The mentioned works mainly concerned the detection of psychological peculiarities of different forms of judgment. Today, following questions were studied: inference of different emotional states on formal logical conclusions, intuitional comprehension of the quantitative material in evaluative judgment, existence of asymmetry phenomenon, the problem of generalization in modern conceptions of forming the concepts. However, earlier and further investigations in fact did not take into the most important property of the inductive thinking, i.e. specificity of probabilistic judgment. In connection with this question Dimitri Uznadze noted:" Logic is interested in objective truth, psychology in its subjective experience, therefore, the problem of judgment's psychology is confidence problem" [1, p 495].

Bounded rationality: For a long time being under the influence of philosophy of logical analysis the investigators of cognitive processes identified everyday thinking with logical reasoning. However, modern psychological research directed on the detection peculiarities of reasoning in different life situations, evidenced the irregularity the above identity. The whole direction was formed, the so-called, research of "bounded rationality," which emerges from the interaction between the cognitive capabilities of the 
subject and the structure of the task environment [2]. Empirical researches concerning psychic determinants of functioning of probabilistic judgments revealed vividly the expressed tendency of ignoring the people of those normative demands which should provide an optimal decision of targeted vital problems and accepted "illogical" choices. Generally speaking, people in real-life conditions do definite conclusions basing on their own needs, beliefs, value sets and aims. This is how their personal rationality is showed.

Probabilistic judgments appear in the conditions of uncertainty, in situations in which there are not strict normative limitations in possible decision. On the basis of multitude of empirical investigations concerning probabilistic judgments then the following conclusion can be formulated: when predicting, people do not use principles of the theory of probabilities, but they use cognitive heuristic rules. Heuristics are simple and often quite approximate strategies for solution of that or other problem These strategies are less accurate than the principle of normative principle and their application does not always make a good choice. However, they have one big advantage: they are simple and not require great mental consumption [3,4,5].

The investigations show that use of heuristic strategies often leads to specific erroneous decisions. The examples of such decisions can be the effects of representativeness, psychological accessibility, ignoring of casual events and word framing alternatives. Using the rule of representativeness, people doing their choice as a certain level of comparison between events, sampling and population in which it kept. The event is more representative, the more it remembered population. Besides, often the reason of erroneous decision is a misunderstanding of the fact that combination of two events (conjunctive judgment) cannot have bigger probability than every event separately. When solving that or other problem, people often are oriented by the strategy of psychological accessibility, according to which the event is more probabilistic and faster stamped in the memory. The application by people of the given heuristic strategy and revealed tendency ignoring significant of casual events (reasons of human mortality) explains why evident and bright descriptions of the events more convincing fore people are than real statistic data. An important factor, which influences on effectiveness of the choice is formulation of the problem. The erroneous decision in this case is determined by the fact that people reveal the tendency of giving different responses on different formulated, but logical identical problems.

Experimental investigation: Basing on the above mentioned theoretical theoretic assumption and considering existing empiric results we conducted the investigation, the aim which consisted in comparative study of influence of prospective and situational sets on probabilistic judgments taking into account feedback factor. Indicated forms of sets are independent variables, the correctness of choice and subjective confidence in it are dependence variables and feedback link presents intermediate variable.

The procedure of the investigation. The participants of the experiment were 144 (75 female and 69 male) students of one private university in Tbilisi. During one 
semester (subject "The work and organizational psychology") two questionnaires were conducted with two-month intervals. Respondents were divided into two experimental and one control group. On first stage of research the respondents of one of the experimental groups were told that some questions in every week questionnaire were as "problem- exercises" and their understanding and given responses would, by all means, contribute to learning of teaching material. Besides, it was underlined that special attention in the teaching program itself is given to fulfillment of this task for a final mark on the studied subject (formation of the prospective set). The respondents of other experimental group were told that the responses on the given questions reflected quickwittedness and skills to solve concrete problems (formation of the situational set). The probabilistic judgments of the participants of the control group were tested twice: the first and third questionnaires. At their each response, the respondent gave a confidence judgment, that is particular answer was correct. The feedback was provided by means of information both experimental groups about the marks received by them during the first questionnaires. We used various examples concerning heuristics of representativeness and accessibility, ignoring of casual events and word formulation effects.

The obtained data. The general result obtained indicates that the difference between indexes of control and situational set's group was statistically insignificant. Note that in the given case the respondents were informed about general mark on the task without explanation of the reason of the obtained mark. Statistically significant difference between control and the group of prospective set was $32 \%(p<0.01)$. This result indicates that respondents with prospective set due to the significant decrease of the quantity of cognitive mistakes and realty improved the quality of probabilistic judgments. The data represented the connection between the feedback and probabilistic judgments indicate that the feedback had statistically significant influence on the group with prospective set, $29 \%(p<0.02)$. Thus, we can say that the feedback is quite effective in case functioning prospective set, the respondents realize the importance of qualitative fulfillment of experimental task in order to achieve a positive result. The obtained results showed a definite impact of the feedback on the degree of respondents' judgment confidence. At the same time, oriented on situation respondents revealed more confidence in their choices than respondents oriented on the future. For example, participants of the first group reflecting in their probabilistic judgments the effect of heuristic representativeness based on the clearness of the perceived information without "any critics." The other tendency is observed in participants of prospective set's group: they are less confident in their own choice and are not tempted to straight adoption on decision.

On the whole, experimental results showed: a) prospective set, compared with situational set, made significant corrective influence on probabilistic judgments; b) medium stage of confidence in compensated decisions was detected with availability 
prospective set, while situational set contributed to the detection of self-confidence concerning quit unsuccessful decisions; c) in the process of functioning of prospective set stimulating impact of feedback on probabilistic judgments was revealed that indicates the importance of cognitive fixation of instrumental dependence between primary and secondary expected results.

Conclusion: The investigation of psychical mechanisms of probabilistic thinking revealed its limited rationality, reflecting of subjective comprehension of one or other problem by people. Obtained empirical results revealed that probabilistic judgments in every day and nonstandard conditions are far from strictly logical construction. However, it does not exclude its possibility of the improvement of their quality as the construction of conditions contributing to reconstruction of erroneous cognitive activity can give a positive result. The following should be taken into account: in the conditions of uncertainty of intentional evaluation of social and personal phenomena, the alternative in fact does not exist. Such phenomena as, for example, the possibility of breaking of the war, the outcome surgical operation, profitable investments are connected with probabilistic judgments. There are no (or very minimal) objective assessment criteria in these cases. The most important is that probabilistic judgment determines targeted behavior and the process of decision making. That is why research of the probabilistic judgments' improvement stays acute problem in the future.

\section{References}

1. Uznadze D. General psychology. Tbilisi, 1998.

2. Simon H. Models of bounded rationality. Cambridge, M.A: MIT Press, 1982.

3. Gigerenzer G., Hertwig R., Pachur T., Heuristics: Foundations of adaptive behavior. N.Y.: Oxford University Press, 2011.

4. Charcviani D., Dzidziguri D., Probability and quantitative estimations in different judgmental contexts. Bull. Georg. Acad. Sci. No.1, 2008.

5. Charcviani D. Evaluative judgment and assimilation effects. Bull. Georg. Acad. Sci, No.1,2014. 